\title{
Over-the-Air Performance Evaluation in Indoor and Outdoor Multipath Propagation Channels of Antenna Diversity Systems inside Anechoic Chamber
}

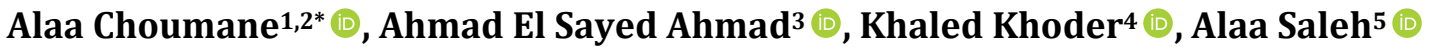 \\ ${ }^{1}$ XLIM, O.S.A. Department-UMR CNRS 6172, University of Limoges, Limoges, France \\ ${ }^{2}$ Faculty of Public Health IV, Lebanese University, Zahle, Lebanon \\ ${ }^{3}$ Faculty of Sciences III, Lebanese University, Tripoli, Lebanon \\ ${ }^{4}$ Department of Innovation and Technology, Azm Institute, Tripoli, Lebanon \\ ${ }^{5}$ Faculty of Technology, Lebanese University, Saida, Lebanon \\ Email: *alaa.choumane@ul.edu.lb, elsayed.ahmad84@gmail.com,kh.khoder@gmail.com
}

How to cite this paper: Choumane, A., El Sayed Ahmad, A., Khoder, K. and Saleh, A. (2020) Over-the-Air Performance Evaluation in Indoor and Outdoor Multipath Propagation Channels of Antenna Diversity Systems inside Anechoic Chamber. Wireless Engineering and Technology, 11, 31-43. https://doi.org/10.4236/wet.2020.114004

Received: September 13, 2020

Accepted: October 14, 2020

Published: October 17, 2020

Copyright $\odot 2020$ by author(s) and Scientific Research Publishing Inc. This work is licensed under the Creative Commons Attribution International License (CC BY 4.0).

http://creativecommons.org/licenses/by/4.0/

\begin{abstract}
With the ever-growing requirement for higher data rates, terminals supporting multiple-input multiple-output (MIMO) technologies are being developed for next-generation. As for wireless device manufacturers, a radio performance evaluation of multi-antenna terminals in desired environments is mandatory before product release. This paper discusses the Over the Air (OTA) performance evaluation of antenna diversity systems in Indoor and Outdoor multi-path propagation channel models inside anechoic chamber, in terms of correlation coefficients and diversity gain (DG). These channel models have been emulated in terms of angles of arrivals (AoA) and cross-polarization ratio (XPR) with Rayleigh fading. For this purpose, SATIMO SG24 measurement system has been used. However, the actual configuration of this system is not able to emulate desired realistic environments. Therefore, an innovative methodology based on the SG24 probes control has been developed. The obtained results in simulations and measurements have shown a good agreement.
\end{abstract}

\section{Keywords}

OTA Testing, Diversity Measurements, Indoor, Outdoor, MIMO, Diversity Parameters

\section{Introduction}

New wireless technologies such as High Speed Downlink Packet Access (HSDPA), 
Long Term Evolution (LTE) and IEEE 802.11n are employing MIMO antenna systems on mobile terminals in order to improve the capacity of data transmission and to overcome the detrimental effects of signal multipath fading when trying to achieve high data throughput in limited-bandwidth channels. Therefore, wireless device manufacturers urgently require standard testing methods that are suitable to evaluate antenna diversity performances before product release. These performances are strongly dependent on antennas properties and the multi-path fading environment. Therefore, a new OTA testing method is required for evaluating antenna diversity performances in realistic multipath propagation environments in order to elaborate efficient multi-antenna terminals.

Since MIMO terminal performances testing in real network is expensive in time consuming, and labor intensive, many OTA methodologies have been adopted in Cellular Telephone Industries Association (CTIA) and 3GPP [1] to evaluate the overall performance of the multi-antenna terminals in a reliable, repeatable, and feasible way in laboratory conditions. Some techniques have been proposed for OTA testing for assessing the performance of antenna diversity terminals in realistic multipath channel [2] [3] [4], like the Reverberation Chamber (RC) method [5] and the multi-probe anechoic chamber (MPAC) method [1]. But the RC is limited to generate an isotropic multi path channel with $0 \mathrm{~dB}$ XPR. On the other hand, the MPAC systems basically consist of a channel emulator and multi probe antennas. However, the main disadvantage with the MPAC is the cost of the setup, since a channel emulator cost increases with the number of antennas.

In this paper, we evaluate the performance of an antenna diversity system using a new test method considerably less complex than other techniques proposed in the literature, in terms of cost-effectiveness. This method is based on replacing the channel emulator with a switch in order to generate a desired realistic Rayleigh channel in terms of AoA and XPR, based on the ignition probabilities of emitting antennas. Two types of propagation models, such as Indoor and Outdoor in terms of AoA, are used for OTA testing in terms of correlation coefficient and diversity gain (DG) computed from the signals received at the different branches of the antenna systems.

\section{New Control Method of SATIMO SG24 Measurement System}

The SATIMO SG24 measurement system as shown in Figure 1 [6], located inside an anechoic chamber of dimensions ( $4 \mathrm{~m} \times 4 \mathrm{~m} \times 4 \mathrm{~m})$, is a real time near field antenna testing system. This system consists of an array of 23 dual-polarized probes encircling a rotation mast used to support the Device Under Test (DUT). These probes are positioned on a $2.4 \mathrm{~m}$ circular arch and spaced by $15^{\circ}$ in elevation. The mast can be rotated azimuthally from $0^{\circ}$ to $360^{\circ}$ and in elevation from $-7.5^{\circ}$ to $7.5^{\circ}$, in order to obtain oversampled data.

The actual configuration of the SG24 measurement system is not able to re- 
produce a desired multipath propagation channel. Wherefore, a new control method of the SG24 probes has been developed. As the material configuration of the SG24 is not able to modify the amplitude and the shift phase of the paths for generating a multi-path channel with Rayleigh fading, a solution consists to displace the DUT horizontally has been proposed.

This method consists in generating a desired multipath propagation channel in terms of angles of arrivals (AoA) at a DUT. It is based on the probes control, used in emitting way, of the SG24 measurement system.

In this method, we consider that at each rotation step of the device under test (centered or moved) a new group of probes is created artificially. So, while rotating the DUT from $0^{\circ}$ to $179^{\circ}$ an artificial spherical distribution of probes is obtained as we illustrate in Figure 2.

However, during the control of the SG24 probes some received paths (angles of arrivals) at the DUT do not exist. Wherefore, in this method, we decompose the space surrounding the device under test in many cells as depicted in Figure 3 . These cells are defined as the discretization of that space in azimuth $(\varphi)$ and elevation $(\theta)$ by a step of $k^{\circ}$ in which we guarantee a possible path.

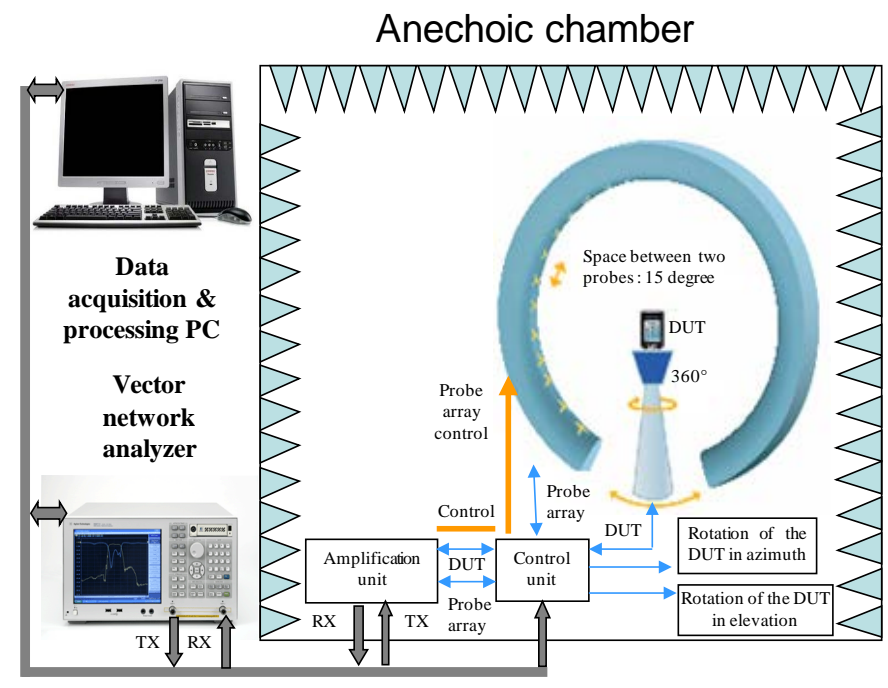

Figure 1. SATIMO SG24 Measurement system Setup.

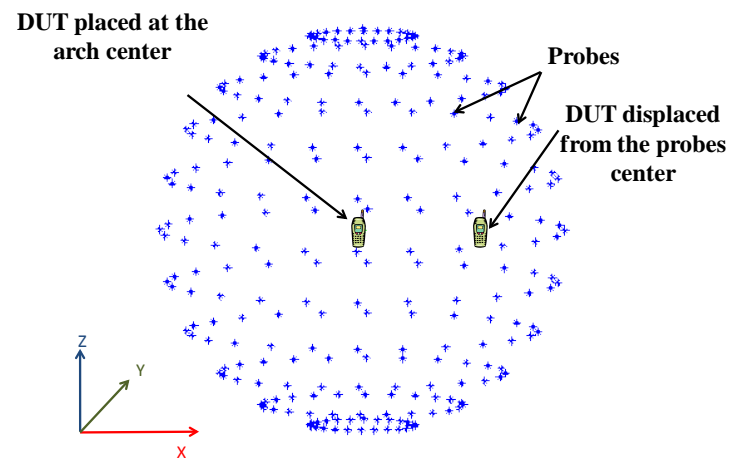

Figure 2. Block diagram of the proposed measurement system. 


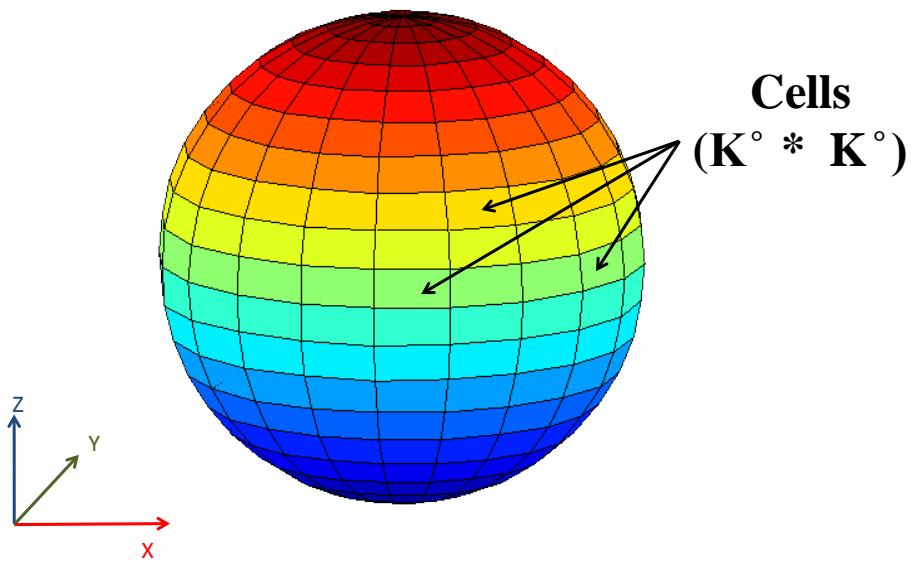

Figure 3. Discretization of space surrounding the DUT in many cells.

In this method, we compute the ignition probabilities of each probe to manage the angles of arrivals at the DUT in order to obtain desired multipath propagation channels. These probabilities are obtained using the law of total probability [7] taking into account that the discretized cells and probes are two family events. In this paragraph, we consider that the probes number of the artificial sphere in elevation $(\theta)$ and in azimuth $(\varphi)$ equal, respectively, to I and J. In addition, the number of discretized cells in elevation $(\theta)$ and azimuth $(\varphi)$ equal, respectively, to $M$ and $N$. The angles of arrivals $(\theta, \varphi)$ of each cell are computed in $(1)$.

$$
\begin{aligned}
& \theta_{n}=\theta_{n}^{\min }+k^{\circ} \\
& \varphi_{m}=\varphi_{m}^{\min }+k^{\circ}
\end{aligned}
$$

where, $\theta_{n}^{\min }, \varphi_{m}^{\min }$ are the minimum AoA of the concerned cell, respectively, in elevation and azimuth.

In order to reproduce a given distribution of the angles of arrivals at the device under test, the ignition probabilities of the SG24 probes can be computed, then, from the equation as listed below:

$$
A=\left[\begin{array}{c}
\text { Pcell }_{11} \\
\vdots \\
\text { Pcell }_{n m} \\
\vdots \\
\text { Pcell }_{N M}
\end{array}\right]=H \cdot B=H \cdot\left[\begin{array}{c}
P\left(\text { Probe }_{11}\right) \\
\vdots \\
P\left(\text { Probe }_{i j}\right) \\
\vdots \\
P\left(\text { Probe }_{I J}\right)
\end{array}\right]
$$

With:

$$
H=\left[\begin{array}{ccccc}
P\left(\text { cell }_{11} \mid \text { Probe }_{11}\right) & \cdots & P\left(\text { cell }_{11} \mid \text { Probe }_{i, j}\right) & \cdots & P\left(\text { cell }_{11} \mid \text { Probe }_{I J}\right) \\
\vdots & \vdots & \vdots & \vdots & \vdots \\
\vdots & \vdots & P\left(\text { cell }_{n m} \mid \text { Probe }_{i, j}\right) & \vdots & \vdots \\
\vdots & \vdots & \vdots & \vdots & \vdots \\
P\left(\text { cell }_{N M} \mid \text { Probe }_{11}\right) & \vdots & \vdots & \vdots & P\left(\text { cell }_{N M} \mid \text { Probe }_{I J}\right)
\end{array}\right]
$$


where:

- The vector $A$ of length equal to $\left(N^{\star} M\right)$ represents the analytical angles of arrivals distribution of the desired multipath propagation channel.

- The vector $B$ of length $\left(I^{\star}\right)$ is the ignition probabilities of the SG24 probes in order to emulate a given AoA distribution at the DUT.

- The Matrix $H$ of size $\left(N^{\star} M, I^{\star}\right)$ is the probability density of a possible path in the cells given a lighted probe of the SG24.

Finally, the ignition probabilities are obtained in resolving the system of linear equations mentioned in (2) according to the QR factorization method with column pivoting.

\section{Channel Characterization and Propagation Models for the Proposed Method}

During the travel between the emitter and the receiver, the transmitted signal is subjected to various electromagnetic phenomena. Wherefore, the multi-path, generated by these phenomena, arrives depolarized with different Angles of Arrivals (AoA) at the device under test. This may affect its performances which vary from a multi-path propagation channel to another. The characterization of these channels can be exemplified by the probability density function of both azimuth $(\theta)$ and elevation $(\varphi)$ Angles of Arrivals of received signals as shown in Figure 4. This function is described as follow:

$$
P(\theta, \varphi)=P(\theta) * P(\varphi)
$$

Many studies, based on AOA distribution measurement in real environments, the so-called field drive testing, have been mentioned that the channels' statistics in "Indoor" and "Outdoor" environments follow a uniform incoming wave distribution in the azimuth plane [8] [9] [10]. On the other hand, the elevation AOA distribution is more complicated than in azimuth since there is a variation in height between the transmitters.

A number of propagation references cases, relies on simulations, have been studied in [11] [12]. In this paper, two reference cases characterized as Indoor and Outdoor propagation models have been chosen as depicted in Table 1 for OTA performance evaluation of an antenna diversity system inside anechoic chamber.

\section{Diversity Measurements}

In this paper, diversity measurements were performed out using antennas systems at the frequency 3.5 GHZ in Indoor and Outdoor multipath propagation channel models. The reference antenna (Figure 5(a)) consists of a linear monopole integrated on a small PCB. The actual diversity system (Figure 5(b)) consists of two identical monopoles spaced by $d=0.14 \lambda$.

We denote, also, that the return loss of the reference and diversity antenna systems at the frequency 3.5 GHZ equal respectively to $-10.37 \mathrm{~dB}$ and -26.682 $\mathrm{dB}$ as depicted in Figure 6. 
Table 1. Propagation models used in this paper.

\begin{tabular}{ccc}
\hline Model & Indoor & Outdoor \\
\hline Elevation/Azimuth & Gaussian/Uniform & Laplacian/Uniform \\
Statistical & $P(\theta)=A * \exp \left[-\frac{\left.\left(\theta-\left[\frac{\pi}{2}-\bar{\theta}\right]\right)^{2}\right]}{2 \sigma^{2}}\right]$ & $P(\theta)=A * \exp \left[-\frac{\sqrt{2}\left[\theta-\left[\frac{\pi}{2}-\bar{\theta}\right]\right]}{\sigma}\right]$ \\
distribution & $P(\varphi)=1$ & $P(\varphi)=1$ \\
Scenario & $X P R=5 \mathrm{~dB}$ & $X P R=1 \mathrm{~dB}$ \\
parameters & $\bar{\theta}=10^{\circ}$ & $\bar{\theta}=20^{\circ}$ \\
& $\sigma=15^{\circ}$ & $\sigma=30^{\circ}$ \\
\hline
\end{tabular}

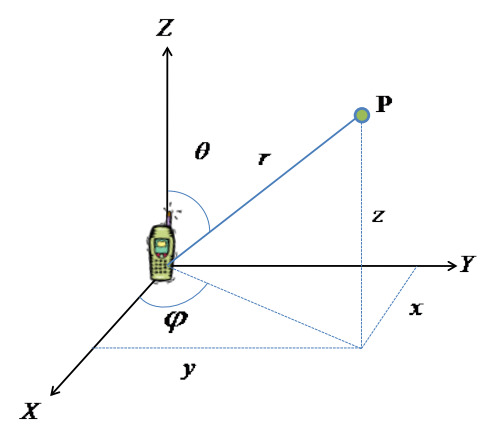

Figure 4. Spherical coordinate representation.

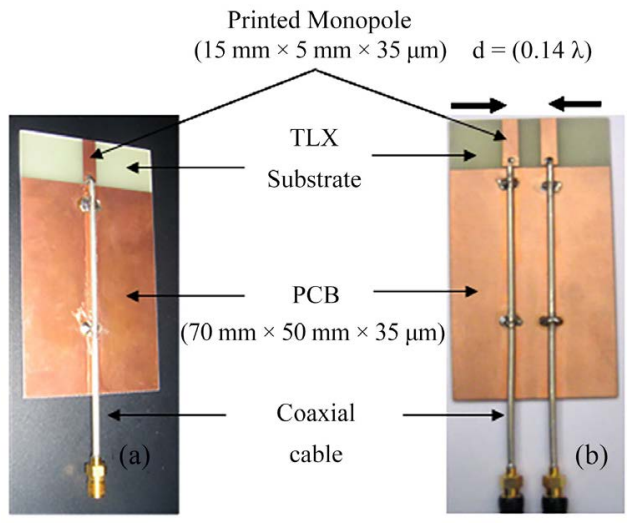

Figure 5. Reference (a) and Diversity system (b).
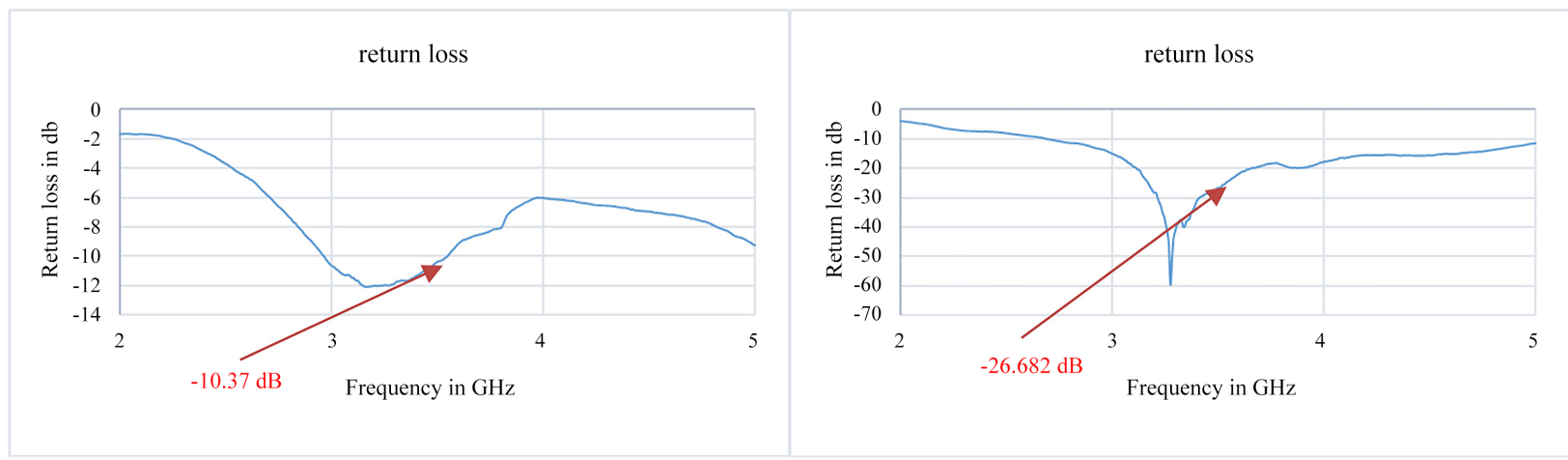

Figure 6. Return loss of Reference antenna and diversity system. 


\subsection{Measurement Setup}

In positioning the device under test at the arch center, the Euclidean distance between SG24 probes and antennas is the same. So, the different paths arrive in a phase which prevents the received signals to be added in a constructive and destructive way in order to obtain the Rayleigh fading. Wherefore, the diversity measurements have been done in placing the antenna systems at 0.7 meter from the arch center (Figure 7) where the Euclidian distance between probes and antennas is different.

In these measurements, a vector network analyzer of 2 ports has been used, where the RX port is connected to the probes and the TX port is related to the DUT. In order to have a good ratio between the angles of arrivals resolution and measurements time, the DUT has been rotated from $0^{\circ}$ to $175^{\circ}$ at $5^{\circ}$ step in azimuth and from $-7^{\circ}$ to $7^{\circ}$ at $1^{\circ}$ step in elevation. In addition, at each position of the DUT 500 samples have been captured.

\subsection{OTA Evaluation Performance of Antenna Diversity Measurement}

In applying the configuration mentioned above, the diversity measurements have been performed using the propagation models mentioned previously in Table 1. A discretization step of $18^{\circ}$ has been computed in order to ensure a possible path in each cell. The probes have been, then, controlled according to the ignition probabilities calculated using our developed method (paragraph 2).

In post processing, the received signals at each position of the antennas systems in elevation have been summed. This procedure has been performed in order to represent $3 \mathrm{D}$ multi-path propagation channels. The obtained signals at each branch of the diversity system are used to evaluate its performances in terms of the correlation coefficient and diversity gain (DG) using the reference antenna system. The DG is deducted from the cumulative distributed function (CDF) plot of the received signals and after a combining according to the diversity combing techniques [13] [14]: Equal Gain Combining (EGC) and Maximum Ratio combining (MRC). These techniques are based on the exploitation of the complex signals received by all the diversity system branches in order to obtain a maximum signal to noise ratio (SNR) at the output of the combiner.

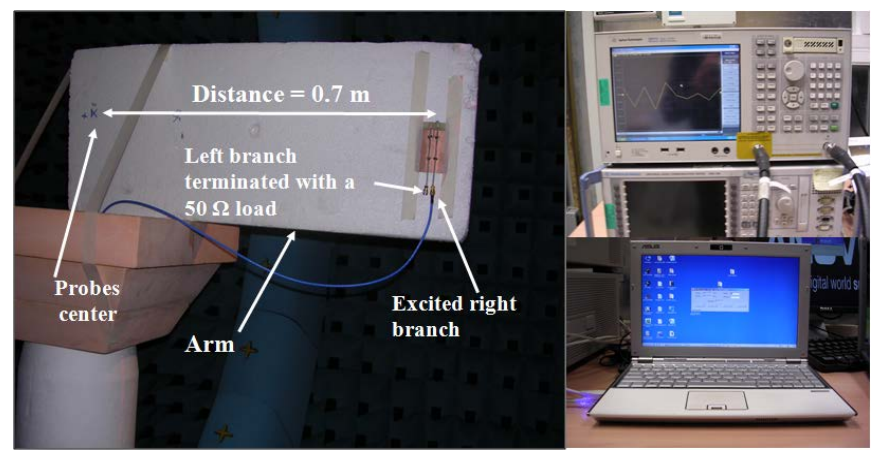

Figure 7. Measurements setup using SATIMO SG24 system. 


\subsubsection{Outdoor Multipath Propagation Model}

Figure 8 shows the analytical and simulated elevation AOA distribution, with respect to the Outdoor model shown in Table 1 , in terms of azimuth planes using the SATIMO SG24 modeling program. In this figure, we see that the analytical and simulated angular dispersion of the arrived waves have a good agreement where it is uniform in azimuth and Laplacian in elevation.

Figure 9 shows the probability density distribution of the fading amplitude of the derived signals, where we see that the simulation and the measurement results have a good compatibility with the Rayleigh distribution. It should be also noted their standard deviations (Sigma) are so close, where their values of about 0.46 .

The cumulative distributed function (CDF) plot of the waves is good indicator to show the diversity gain performance in a fading environment. Figure 10 and Figure 11 show, respectively, the CDF plot of simulated and measured signals powers provided for each diversity branch and by applying the diversity combing methods EGC and MRC.

In these figures, we denote that the different curves are very similar. From these curves, we have deduced the diversity gain (DG) at $1 \%$ probability, where it presents $10.4 \mathrm{~dB}$ in simulation and measurement according to the MRC combing method.

The envelope correlation coefficients between the signals (measured and simulated) received by the two branches of the diversity system have been computed using its expression mentioned in [14]. A consolidated summary of the different simulated and measured diversity parameters is depicted in Table 2, where we see that they have a good agreement.

Table 2. Comparison between simulated and measurements results in applying Outdoor model.

\begin{tabular}{cccc}
\hline & $\begin{array}{c}\text { Envelope correlation } \\
\text { coefficient }\end{array}$ & $\begin{array}{c}\text { DG at } 1 \% \text { probability } \\
(d B) / E G C\end{array}$ & $\begin{array}{c}\text { DG at } 1 \% \text { probability } \\
(d B) / M R C\end{array}$ \\
\hline SATIMO Measurement & 0.1 & 10 & 10.4 \\
SATIMO Simulation & 0.09 & 10 & 10.4 \\
\hline
\end{tabular}
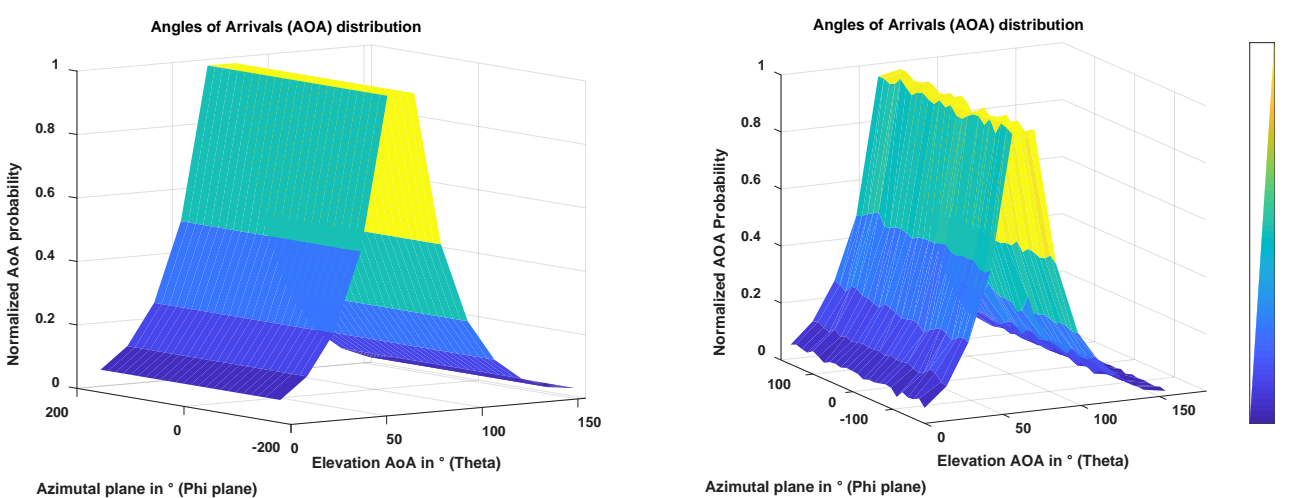

Figure 8. Analytical and simulated elevation AOA distribution in terms of azimuth planes applying the Outdoor model. 


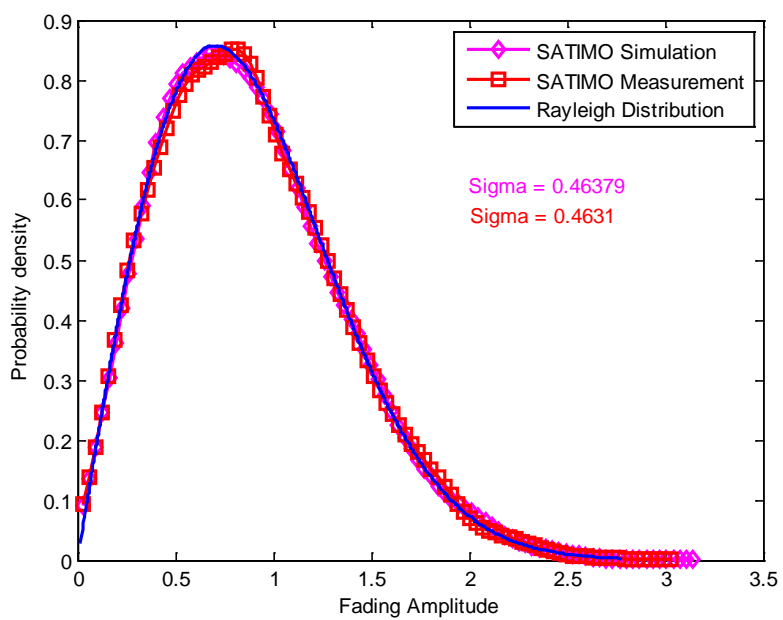

Figure 9. Fading amplitude of the received signals in applying Oudoor model.

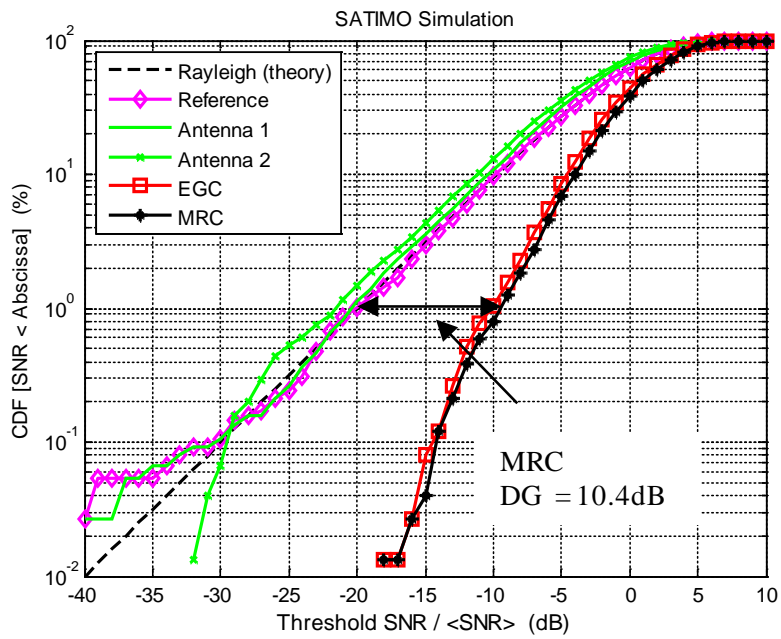

Figure 10. CDF plot of the simulated signal powers at each branch of antenna systems using SATIMO SG24 modelling program, and after diversity combining: EGC and MRC.

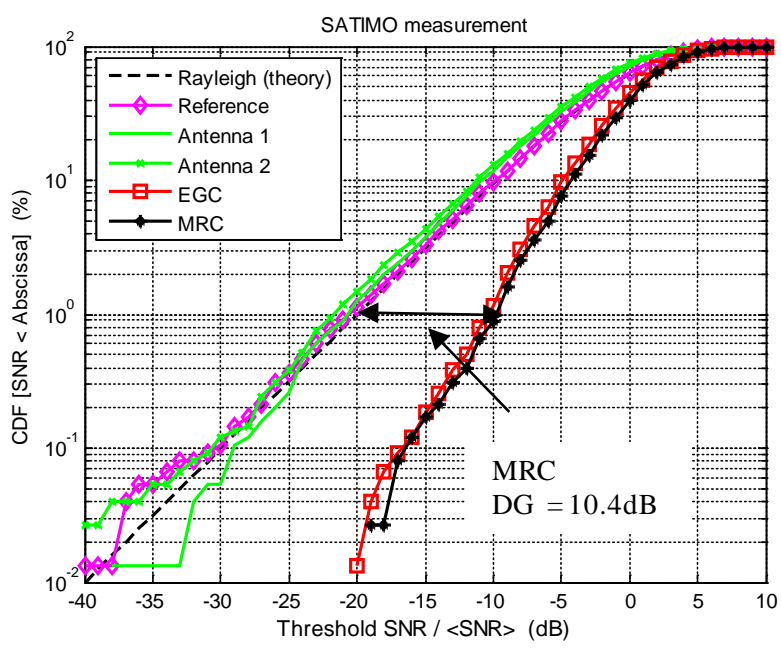

Figure 11. CDF plot of the measured signal powers at each branch of antenna systems using SATIMO SG24 measurements system, and after diversity combining: EGC and MRC. 


\subsubsection{Indoor Multipath Propagation Model}

This model represents an Indoor environment where its characterization is listed previously in this paper. Figure 12 shows analytical and simulated elevation angular dispersion of arrived waves at the DUT, with respect to the Indoor model mentioned in Table 1, in terms of azimuth planes using the SATIMO SG24 modeling program. In this figure, we denote that the analytical and simulated AOA distributions are similar whereas that is uniform in azimuth and Gaussian in elevation.

Figure 13 illustrates the probability densities of the fading amplitude of simulated and measured received signals, where we denote their compatibility with the Rayleigh distribution. In addition, the standards deviations of these signals are very close, where they show a value of about 0.46 .

The performance of the antenna diversity systems can be evaluated in terms of the diversity parameters (correlation coefficient, diversity gain). These parameters can be derived from the received signals at the different branches of antennas systems. The cumulative distributed function $(\mathrm{CDF})$ plot of these signals is a good indicator to deduce the diversity gain in a fading environment. The correlation coefficients have been evaluated, and they have shown a good agreement. The CDF plot of the simulated and the measured signals using the reference and the diversity systems have been performed as illustrated in Figure 14 and Figure 15 These figures show also the CDF combinations of these signals, by applying the diversity combing methods: EGC and MRC.

In observing these figures above, we can deduce the diversity gain. It shows at $1 \%$ probability a value of $10 \mathrm{~dB}$ and $9.7 \mathrm{~dB}$, respectively, in simulation and measurement using the MRC combining method.

The envelope correlation coefficients between the signals (measured and simulated) received by the two branches of the diversity system have been computed. The simulated and measured diversity parameter results have been shown in a good agreement as we see in Table 3.

\section{Conclusions}

In this paper, the performance of an antenna diversity system has been evaluated in indoor and outdoor multipath propagation channel models in terms of correlation coefficient and diversity gain using an antenna reference system. These channel models have been emulated using SATIMO SG24 measurements system in terms of angles arrivals (AoA) and cross-polarization ratio (XPR) with Rayleigh fading. Basically, this system is not able to generate a multipath propagation channel. For this purpose, a new test method based on the control of the SG24 probes has been developed. The obtained results in simulation and measurement results have shown a good agreement.

We denote that the performances of antenna diversity systems were evaluated using a passive measurement in terms of diversity parameters. This technique consists of calculating these parameters from the transmission coefficients (Sij) between transmission and reception using a vector network analyzer. However, 

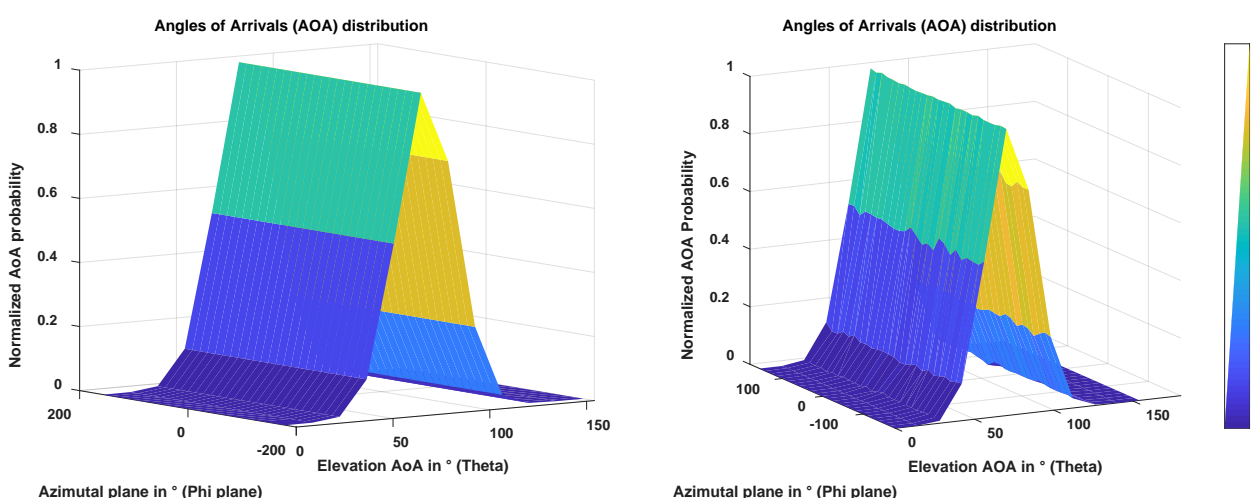

Figure 12. Analytical and simulated elevation AOA distribution in terms of azimuth planes applying the Indoor model.

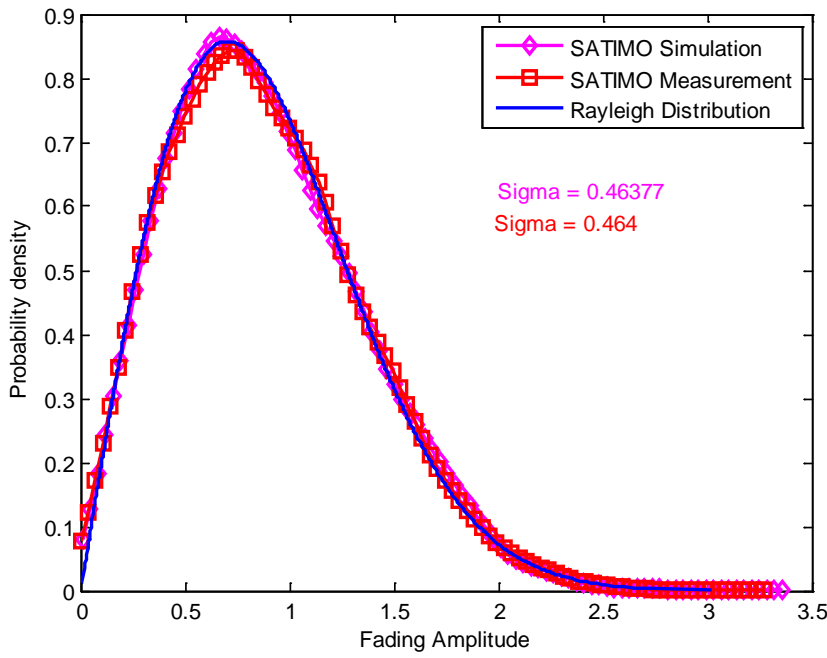

Figure 13. Fading amplitude of the received signals in applying Indoor model.

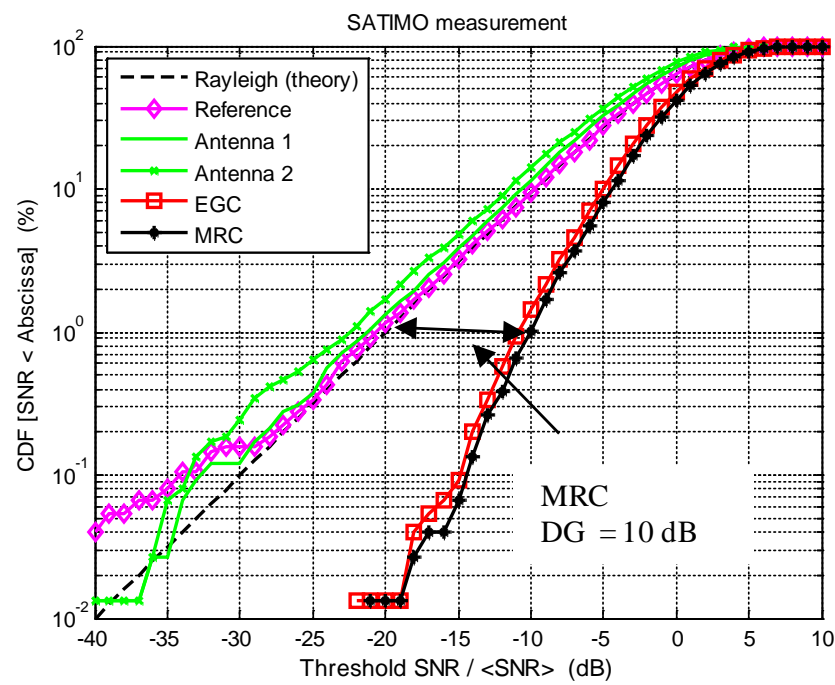

Figure 14. CDF plot of the measured signal powers at each branch of antenna systems using SATIMO SG24 measurements system, and after diversity combining: EGC and MRC. 


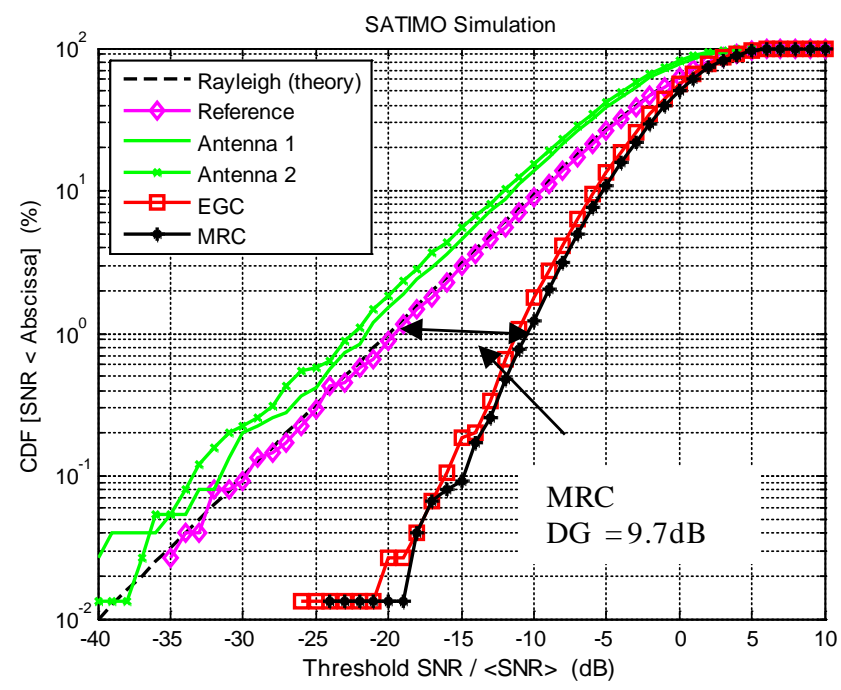

Figure 15. CDF plot of the simulated signal powers at each branch of antenna systems using SATIMO SG24 modelling program, and after diversity combining: EGC and MRC.

Table 3. Comparison between simulated and measurements results in applying Indoor model.

\begin{tabular}{cccc}
\hline & $\begin{array}{c}\text { Envelope correlation } \\
\text { coefficient }\end{array}$ & $\begin{array}{c}\text { DG at } 1 \% \text { probability } \\
(d B) / E G C\end{array}$ & $\begin{array}{c}\text { DG at } 1 \% \text { probability } \\
(d B) / M R C\end{array}$ \\
\hline SATIMO Measurement & 0.14 & 9.5 & 10 \\
SATIMO Simulation & 0.14 & 9.3 & 9.7 \\
\hline
\end{tabular}

this technique does not make it possible to evaluate the performance of mobile terminals in a real way. Therefore, the future work of this paper will be focused on the active measurement technique. This technique makes it possible to evaluate the overall MIMO performances taking into account the antennas but also the complete transmission chain as a function of the binary error rate (BER: Binary Error Ratio), symbol error (SER: Symbol Error Ratio) and frame error rate (FER: Frame Error Ratio).

\section{Conflicts of Interest}

The authors declare no conflicts of interest regarding the publication of this paper.

\section{References}

[1] 3GPP (2013) Verification of Radiated Multi-Antenna Reception Performance of User Equipment. Tech. Rep. 37.977 V1.0.0.

[2] Honda, K., Narukawa, N. and Ogawa, K. (2019) OTA Experiments on the Angular Accuracy of a Phase Monopulse AOA Antenna Using the Mean IQ-Value Method in a Rician Fading Channel. 2019 URSI International Symposium on Electromagnetic Theory (EMTS), San Diego, 27-31 May 2019, 1-4. https://doi.org/10.23919/URSI-EMTS.2019.8931523

[3] Kyosti, P., Kolu, J., Nuutinen, J.-P. and Falck, M. (2008) OTA Testing for Multian- 
tenna Terminals. TD(08)670,COST2100, Lille, France, 6-8 Octobre 2008.

[4] Yamamoto, A., Sakata, T., Hayashi, T., Ogawa, K., Nielsen, J.O., Pedersen, G.F., Takada, J. and Sakaguchi, K. (2010) Effectiveness of a Fading Emulator in Evaluating the Performance of MIMO Systems by Comparaison with a Propagation Test. The 4rd European Conference on Antennas and Propagation EuCAP 2010, Barcelona, 12-16 April 2010, 1-5.

[5] Rosengren, K. and Kildal, P.-S. (2005) Radiation Efficiency, Correlation, Diversity Gain and Capacity of a Six-Monopole Antenna Array for a MIMO System: Theory, Simulation and Measurement in Reverberation Chamber. IEE Proceedings-Microwaves Antennas and Propagation, 152, 7-16.

[6] https://www.mvg-world.com/sites/default/files/2020-05/Datasheet Antenna\%20Me asurement SG 24_05 20_BD 7.pdf

[7] Bertsekas, D.P. and Tsitsiklis, J.N. (2000) Introduction to Probability. Athena Scientific, Belmont, Massachusetts.

[8] Kalliola, K., Laitinen, H., Sulonen, K., Vuokko, L. and Vainikainen, P. (2001) Directional Radio Channel Measurements At Mobile Station in Different Radio Mobile Environments at $2.15 \mathrm{GHZ}$. Proceeding of the 4th European Personal Mobile Communications Conference, Vienna, 20-22 February 2001.

[9] Kuchar, K., Rossi, J.-P. and Bonek, E. (2000) Directional Macro-Cell Channel Characterization from Urban Measurements. IEEE Transaction on Antenna and Propagation, 48, 137-146. https://doi.org/10.1109/8.833062

[10] Spencer, Q., Rice, M., Jeffs, B. and Jensen, M. (1997) A Statistical Model for Angle of Arrival in Indoor Multipath Propagation. 1997 IEEE 47 th Vehicular Technology Conference. Technology in Motion, Phoenix, AZ, 4-7 May 1997, 1415-1419.

[11] Fujimoto, K. (2008) Mobile Antenna Systems Handbook. Third Edition, Artech House Inc., Massachusetts, USA.

[12] Planic, V. (2004) Antenna Diversity Studies and Evaluation. Master Thesis, LUNDS University in Cooperation with Ericsson Mobile Communications AB, Lund, Sweden.

[13] Saunders, S.R. and Zavala, A.A. (2007) Antennas and Propagation for Wireless Communication Systems. John Wiley \& Sons, Inc., New York.

[14] Khaleghi, A., Azoulay, A. and Bolomey, J.C. (2005) Diversity Techniques with Dipole Antennas Indoor Multi path Propagation. 16th Annual IEEE International Syposium on Personal Indoor and Mobile Radio Communication (PIMRC), Berlin, 11-14 September 2005, 669-673. 